\title{
ENRIQUECIMIENTO DE TAREAS EN LA FORMACIÓN INICIAL DEL GRADO DE PRIMARIA PARA ATENDER A LOS PROGRAMAS DE PROFUNDIZACIÓN
}

\author{
Rafael Ramírez Uclés \\ Universidad de Granada
}

\begin{abstract}
RESUMEN: Las normativas curriculares recientes resaltan la necesidad de atender las altas capacidades, particularmente al estudiantado que presenta talento matemático. En este trabajo se ejemplifica una tarea de formación para que los futuros maestros diseñen tareas de enriquecimiento en un programa de profundización. Esta propuesta se fundamenta en el análisis didáctico como herramienta de planificación de la enseñanza. A partir de la caracterización del talento matemático y la noción de tarea rica, se establecen algunas pautas para que sea el estudiantado del Grado de Primaria el que analice y diseñe tareas para el alumnado especialmente motivado o que presente altas capacidades matemáticas.
\end{abstract}

PALABRAS CLAVE: análisis didáctico, formación inicial de maestros, talento matemático, tarea rica, programas de profundización.

\section{ENRICHMENT OF TASKS IN INITIAL FORMATION OF THE BACHELOR'S DEGREE IN PRIMARY EDUCATION TO ATTEND DEEPENING COURSES}

\footnotetext{
ABSTRACT: Recent curricular regulations highlight the need to attend to high abilities, particularly students with mathematical talent. This proposal shows a training task example for future teachers to design enrichment tasks in an enhancement program. This work is based on didactic analysis as a teaching planning tool. Based on the characterization of mathematical talent and the notion of rich activities, some guidelines are established for the Primary Grade student analyze and design tasks for specially motivated or mathematical talented students in Primary Grade.
} 
KEYWORDS: Didactic analysis, enrichment program, preservice teachers, mathematical talent, rich tasks

Recibido: 01/02/2021

Aceptado: 09/03/2021

Correspondencia: Rafael Ramírez Uclés, Facultad de Ciencias de la Educación, Universidad de Granada, Campus Universitario de Cartuja,18071 Granada. Email: rramirez@ugr.es

\section{INTRODUCCIÓN}

Recientemente, el 15 de enero de 2021, la Consejería de Educación y Deporte reguló determinados aspectos de la atención a la diversidad en la orden que desarrolla el currículo correspondiente a la etapa de Educación Primaria en la Comunidad Autónoma de Andalucía (Junta de Andalucía, 2021). Dentro de los programas de atención a la diversidad, se establece

Asimismo, los centros docentes podrán establecer programas de profundización para el alumnado especialmente motivado para el aprendizaje o para aquel que presente altas capacidades intelectuales (p. 11).

Destacamos dos ideas de estos programas. Por un lado, tienen como objetivo dar respuesta a las necesidades tanto del alumnado altamente motivado para el aprendizaje como para el que presenta altas capacidades intelectuales. Por otro lado, consisten en un enriquecimiento de los contenidos del currículo ordinario con actividades que supongan el desarrollo de tareas o proyectos de investigación que estimulen la creatividad y la motivación del alumnado.

En este trabajo presentamos algunas ideas que puedan servir de apoyo en la formación inicial del futuro maestro de Primaria (FMP) para abordar estas tareas de enriquecimiento en la clase de matemáticas. Para ello, partiremos inicialmente de la caracterización de los estudiantes con altas capacidades matemáticas y fundamentaremos nuestra propuesta en el análisis didáctico como referente teórico para sustentar la planificación (Rico, 1997a, 1997b; Rico et al., 2013). Nuestra propuesta se focalizará en aportar estrategias al FMP para diseñar y modificar tareas ricas (Stein et al., 2000).

\section{Talento matemático o alta CAPACidAd matemÁtica}

Especialmente en edades tempranas, la identificación de un estudiante como talento matemático o alta capacidad matemática viene sustentada en la obtención de puntuaciones correspondientes a percentiles elevados en test estandarizados. En cambio, desde la Educación Matemática se han propuesto diferentes listados de características específicas para que sea el propio maestro el que nomine al estudiante. Si bien el listado y descripción de la caracterización del talento se ha abordado de manera extensa (entre otros, Greenes, 1981; Krutetskii, 1976; Miller, 1990), consideramos que estas descripciones podrían resultar no del todo operativas para que el docente identifique a los estudiantes y diseñe los programas de profundización. Por ejemplo, el contexto de 
la resolución de problemas es uno que se ha destacado como útil para identificar estas características (Nierderer et al., 2003). Al resolverlos, el maestro debe valorar, entre otras pautas: si el estudiante comprende con mucha facilidad la información que se le proporciona; cuando está interesado, aprende con facilidad y rapidez; entiende ideas abstractas y conceptos complicados para su edad; es innovador y original planteando y resolviendo problemas; muestra un rendimiento especialmente bueno en una o más áreas y posee habilidad para el cálculo mental (Piñeiro et al., 2017). Hay características, como alguna de las señaladas por Freiman (2006) descritas en estas edades (cambia fácilmente de una estrategia a otra, localiza la clave de los problemas, mantiene bajo control los problemas y su resolución o piensa de modo crítico) que plantean al maestro un reto para ser identificadas y, quizás más relevante, para ser atendidas. Más allá de la creatividad, motivación e iniciativa personal, consideramos que el maestro necesita una serie de estrategias para planificar y diseñar buenas prácticas docentes en este alumnado. En este sentido, presentamos nuestra propuesta basada en el análisis didáctico.

\section{El anÁlisis didáctico como planificación de la enseñanza en Educación Primaria}

El análisis didáctico es definido como "el procedimiento con el que es posible explorar, profundizar y trabajar con los diferentes y múltiples significados del contenido matemático escolar, para efectos de diseñar, Ilevar a la práctica y evaluar actividades de enseñanza y aprendizaje" (Gómez, 2007, pp. 18-19). Para la formación inicial, se ha presentado este modelo en propuestas formativas en manuales destinados tanto a la Educación Primaria (Flores y Rico, 2015) como para la Educación Secundaria (Rico y Moreno, 2016), cuyo objetivo es que el FMP entienda y utilice el conocimiento didáctico del contenido matemático escolar para diseñar tareas escolares y unidades didácticas de las matemáticas. Rico (2015) establece la conexión entre el conocimiento matemático escolar y el conocimiento didáctico de las matemáticas escolares al desarrollar el análisis didáctico, que incluye cuatro análisis (de contenido, cognitivo, de instrucción y evaluativo).

Vamos a describir brevemente estos cuatro análisis, para focalizarnos posteriormente en el apartado relativo al diseño de tareas ricas. Inicialmente, se realiza un análisis de contenido para concretar el aspecto cultural/conceptual y estudiar el significado de los contenidos matemáticos escolares. Este análisis aborda las estructuras conceptuales, la evolución histórica, sistemas de representación y modelos y análisis fenomenológico. Se determinan así los significados prioritarios para el aprendizaje y la enseñanza del contenido matemático.

A partir de los focos del análisis anterior, el FMP realiza el análisis cognitivo, identificando las posibilidades y obstáculos de aprendizaje del estudiante y establece las finalidades de aprendizaje formuladas en forma de objetivos y competencias. Además, realiza una búsqueda de los errores y dificultades de dicho tema.

En el posterior análisis de instrucción es donde se analizan, diseñan y secuencian las tareas matemáticas escolares. Se buscan tareas matemáticas escolares significativas, examinando los materiales y las prácticas más adecuadas para alcanzar los objetivos de aprendizaje. La síntesis de este proceso es el diseño de la unidad didáctica. 
Finalmente, en el análisis evaluativo se proponen instrumentos, criterios y formas de poner en práctica la evaluación para apreciar los logros del estudiantado. A modo de síntesis, presentamos en la tabla 1 las siguientes categorías del análisis didáctico, destacando para este trabajo los organizadores curriculares del análisis de instrucción.

Tabla 1. Categorías para el análisis didáctico. Extraído de Rico (2016)

\begin{tabular}{llll}
\hline \multicolumn{4}{c}{ Dimensiones } \\
$\begin{array}{l}\text { Primera: cultural- } \\
\text { conceptual }\end{array}$ & Segunda: cognitiva & $\begin{array}{l}\text { Tercera: } \\
\text { ético-normativa }\end{array}$ & Cuarta: social \\
\hline $\begin{array}{l}\text { Análisis de los } \\
\text { significados }\end{array}$ & Análisis cognitivo análisis & $\begin{array}{l}\text { Análisis de } \\
\text { instrucción }\end{array}$ & Análisis evaluativo \\
\hline \multicolumn{1}{l}{$\begin{array}{l}\text { Significado de los } \\
\text { contenidos matemáticos } \\
\text { escolares }\end{array}$} & Condiciones de estudio & $\begin{array}{l}\text { Planificación e } \\
\text { implementación } \\
\text { yorientación } \\
\text { del aprendizaje la enseñanza de } \\
\text { matemático escolar }\end{array}$ & $\begin{array}{l}\text { Aprendizajes } \\
\text { las matemáticas } \\
\text { lanformación, } \\
\text { valoración y toma } \\
\text { de decisiones }\end{array}$ \\
\hline
\end{tabular}

Organizadores curriculares o categorías para el análisis de cada dimensión

\begin{tabular}{|c|c|c|c|}
\hline $\begin{array}{l}\text { 1. Estructura conceptual } \\
\text { 2. Sistemas de } \\
\text { representación } \\
\text { 3. Sentidos y modos } \\
\text { de uso }\end{array}$ & $\begin{array}{l}\text { 1. Expectativas de } \\
\text { aprendizaje } \\
\text { 2. Limitaciones } \\
\text { 3. Oportunidades de } \\
\text { aprendizaje }\end{array}$ & $\begin{array}{l}\text { 1. Tareas y } \\
\text { secuencias } \\
\text { 2. Organización } \\
\text { del trabajo en el } \\
\text { aula } \\
\text { 3. Materiales y } \\
\text { recursos }\end{array}$ & $\begin{array}{l}\text { 1. Modalidades y } \\
\text { diseño } \\
\text { 2. Intervención y } \\
\text { toma de decisiones } \\
\text { 3. Indicadores de } \\
\text { calidad }\end{array}$ \\
\hline
\end{tabular}

Contenidos didácticos o componentes de los organizadores para el análisis de cada contenido matemático

\begin{tabular}{|c|c|c|c|}
\hline $\begin{array}{l}\text { 1. Propiedades } \\
\text { formales, funcionalidad } \\
\text { cognitiva-actitudes } \\
\text { emocionales, morales } \\
\text { y éticas } \\
2 \text {. Representaciones } \\
\text { simbólicas, gráficas, } \\
\text { numéricas } \\
\text { 3. Términos, contextos, } \\
\text { fenómenos, situaciones }\end{array}$ & $\begin{array}{l}\text { 1. Objetivos, } \\
\text { competencias, } \\
\text { compromisos } \\
\text { 2. Errores, dificultades, } \\
\text { bloqueos } \\
\text { 3. Condiciones, } \\
\text { demandas, retos }\end{array}$ & $\begin{array}{l}\text { 1. Variables de } \\
\text { tarea, funciones } \\
\text { 2. Complejidad, } \\
\text { creatividad, } \\
\text { organización } \\
\text { 3. Características, } \\
\text { tipos y usos }\end{array}$ & $\begin{array}{l}\text { 1. Funciones, } \\
\text { normativa, } \\
\text { momentos } \\
\text { 2. Criterios, } \\
\text { instrumentos, } \\
\text { rendimiento } \\
\text { 3. Valoración } \\
\text { estratégica, estudios } \\
\text { comparativos }\end{array}$ \\
\hline \multicolumn{4}{|c|}{ Síntesis } \\
\hline $\begin{array}{l}\text { Significados prioritarios } \\
\text { para su aprendizaje y } \\
\text { enseñanza }\end{array}$ & $\begin{array}{l}\text { Estructura de cada } \\
\text { tarea matemática } \\
\text { relativa al aprendizaje } \\
\text { esperado }\end{array}$ & $\begin{array}{l}\text { Organización } \\
\text { de la enseñanza } \\
\text { mediante unidades } \\
\text { didácticas }\end{array}$ & $\begin{array}{l}\text { Logros y calidad } \\
\text { de los aprendizajes } \\
\text { alcanzados }\end{array}$ \\
\hline
\end{tabular}


El análisis didáctico permite al FMP realizar un proceso de profundización tanto en el conocimiento matemático como en su conocimiento didáctico de un tema. El resultado final es la elaboración de la unidad didáctica, entendida no como un proceso burocrático sino como instrumento para planificar la enseñanza. En nuestro caso, este proceso se lleva a cabo en la asignatura Diseño y desarrollo del currículum de Educación Primaria, correspondiente al tercer curso de Grado de Educación Primaria (Ramírez, 2021).

Con la intencionalidad de aportar estrategias para el diseño de programas de profundización, matizamos el apartado relativo a tareas para dar respuesta al enriquecimiento curricular, que consiste en enseñar nuevos contenidos que no estén cubiertos en el currículo oficial o trabajar en un nivel de mayor profundidad, tratando los temas con un nivel mayor de abstracción y complejidad. No consiste en avanzar sobre contenidos de cursos posteriores, sino de ampliar la estructura de los temas, promover el uso de la investigación o del pensamiento creativo y de explorar la lógica interna de este y sus relaciones con otras áreas de conocimiento. Si bien estas ideas son más susceptibles de Ilevarlas a cabo en Educación Secundaria (Blanco et al., 2004), vamos a matizar esta idea de riqueza en tareas escolares de Educación Primaria.

\section{TAREAS RICAS}

El papel de las tareas cubre un papel destacado en la formación de los docentes de matemáticas (Clarke et al., 2009; Tirosh y Woods, 2008; entre otros). Hablamos de tareas matemáticas escolares, entendidas como un segmento de la actividad de clase que se dedica al desarrollo de una idea matemática particular (Stein y Smith, 1998). Entendemos la tarea más allá de la actividad propuesta al estudiante pues incluye toda la "escenografía" que conlleva, desde el diseño hasta la gestión de su implementación en el aula, considerando las intervenciones e interacciones del profesor y los estudiantes, los materiales y todo lo que ocurre en ese segmento de clase.

Cuando una tarea matemática escolar se incluye en la formación de docentes, se aborda desde una perspectiva diferente. Los FMP tienen que aprender las matemáticas (Aguayo, 2018), pero también se incluyen cuestiones asociadas a la didáctica de la matemática (Liljedahl et al., 2007).

La idea de riqueza en las tareas ha sido tratada extensamente (por ejemplo, Burkhardt y Swan, 2013; Grootenboer, 2009; Lingard et al., 2001), pero hay poca investigación que describa cómo ayudar a los maestros a diseñar tareas (Stein et al., 2000). Esos autores sugieren que los profesores necesitan usar tareas ricas para mantener la demanda cognitiva favoreciendo que los estudiantes hagan generalizaciones y justifiquen sus soluciones. Definen una tarea rica como aquella que es compleja, no algorítmica y no rutinaria, lo que permite múltiples estrategias y representaciones y varias rutas hacia una solución. Ofrece a los estudiantes oportunidades para generalizar y justificar que van más allá de encontrar la respuesta, incluye la justificación de la estrategia o razonamiento utilizado para llegar a una respuesta o una explicación de por qué este enfoque particular es válido. También Good (2013) considera que debe: invitar a los estudiantes a practicar matemáticas, ser un desafío apropiado para todos los estudiantes, ser matemáticamente interesantes y proporcionar al profesor datos útiles. 
De la caracterización de otros autores destacamos la idea de que las tareas ricas deben ser accesibles pero desafiantes, que recompensen el razonamiento en lugar de los resultados, que utilicen contextos auténticos para comprender mejor el mundo exterior y que brinde oportunidades a los estudiantes para tomar decisiones (Burkhardt y Swan, 2013). Estas características adquieren matices cuando se abordan en edades tempranas, pero sigue resaltándose la necesidad de que las tareas exploren y desarrollen ideas matemáticas, que se refieran a situaciones familiares del alumnado, que suponga un reto que implique diferentes representaciones, que admita múltiples soluciones y que sea comprensible para que el estudiante esté convencido de que pueda resolverla y que sepa cuando ha encontrado la solución (Ramírez et al., 2018).

Nuestro planteamiento enfatiza que el FMP resuelva tareas escolares ricas para posteriormente analizar el potencial didáctico con los referentes teóricos presentados. En este análisis se enfatizan las tareas de formación que fomenten la enseñanza con sentido (Ruiz-Hidalgo et al., 2019):

- a través de profundizar en el conocimiento del significado de los conceptos matemáticos escolares, que se puede concretar en identificar y organizar situaciones, contextos, fenómenos y modos de uso de cada concepto

- mediante el desarrollo del conocimiento matemático con sentido de los estudiantes para profesor

- como provisión de herramientas para llevar a cabo una enseñanza significativa en su vida profesional futura (p. 123).

Así, consideramos necesario que el FMP resuelva tareas ricas, las analice atendiendo a los organizadores curriculares del análisis didáctico para, finalmente, hacer sus propios diseños justificados según los referentes teóricos (Figura 1). En este proceso se espera que adquiera un mayor conocimiento matemático y conocimiento didáctico del contenido, cubriendo las dos dimensiones del modelo del conocimiento del profesor (Ball et al., 2008).

Figura 1. Tareas ricas de formación

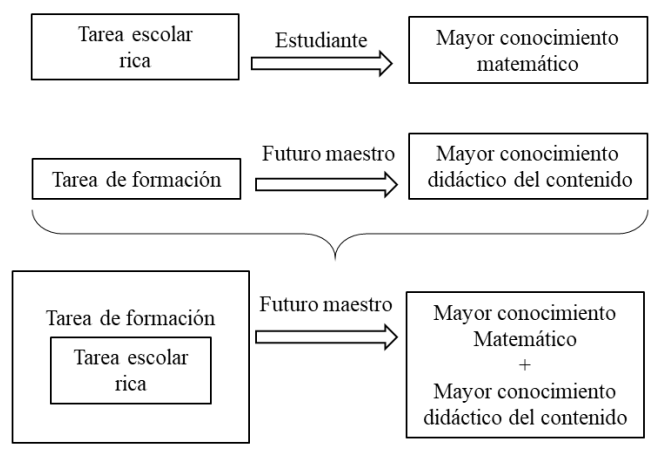

Ejemplificaremos este proceso con una tarea formativa para diseñar una tarea de enriquecimiento. 


\section{EJEMPLO DE TAREA FORMATIVA PARA EL DISEÑO DE TAREAS DE PROFUNDIZACIÓN}

Presentamos un esquema en la figura 2 de nuestra propuesta elaborada a través de los referentes teóricos utilizados.

Figura 2. Proceso de diseño de una tarea de profundización

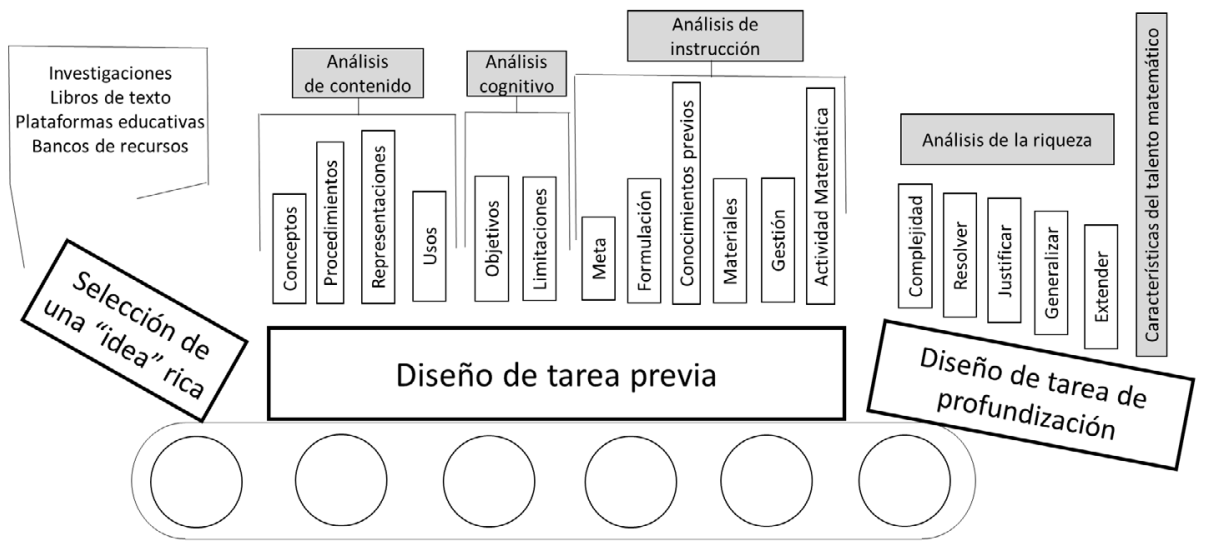

\subsection{Selección de una "idea rica"}

El punto de partida puede ser cualquier tarea escolar de las trabajadas previamente en el análisis de instrucción o de las propuestas en cualquier libro de texto. Pero es recomendable partir de ideas que tienen potencial para convertirse en tareas ricas. En este ejemplo, partiremos de una tarea propuesta en la página de NRICH (https://nrich. maths.org/), que es una reconocida publicación de la Universidad de Cambridge para seleccionar actividades de todos los niveles educativos y organizadas por tópicos, lo que facilita encontrar tareas para un tema específico.

Esta plataforma tiene un apartado (Primary curriculum mapping) que propone tareas para contenidos curriculares específicos. Por ejemplo, en la Unidad didáctica de Suma con Números Naturales, si seleccionamos el tópico Números y Valor posicional, en Tercero de Primaria y más concretamente "para resolver problemas numéricos y practicar problemas que requieran estas ideas", nos aparecen varias propuestas. Seleccionamos Magic Vs (https://nrich.maths.org/6274?utm_source=primary-map) que además viene catalogada como adecuada para la clase completa.

La actividad para estudiantes de Primaria viene formulada de la siguiente manera:

Coloca cada uno de los números del 1 al 5 en la forma de $V$ como a continuación para que los dos brazos de la $V$ sumen la misma cantidad (figura 3 ).

¿Cuántas posibilidades diferentes hay? ¿Qué observas sobre las soluciones que encuentras? ¿Puedes explicar lo que ves? ¿Puedes convencer a alguien de que tienes todas las soluciones? ¿Qué ocurre si usamos los números del 2 al 6? ¿Del 12 al 16? ¿Del 37 a 41 ? ¿de 103 a 107? ¿Qué puedes descubrir sobre una $V$ que tiene brazos de longitud 4 usando los números del 1 al 7 ? 
Figura 3. Disposición del Magic Vs

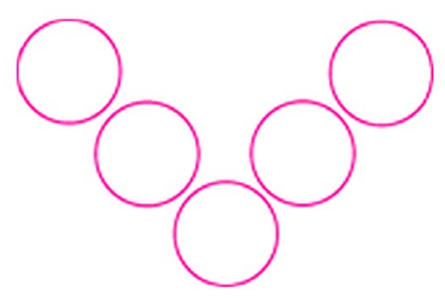

La página tiene un apartado con pistas para ir abordando el problema, que les puede ser muy útil para la gestión de la actividad en el aula:

Intenta poner los números en la $V$ y luego sumar cada brazo. ¿Puedes ajustar la disposición ligeramente para que los totales sean iguales? ¿Qué notas sobre el número en la base de la $V$ en todas tus $V$ mágicas?

También en el apartado de recursos para el profesor le da pautas de los objetivos que persigue este problema e incluso vídeos explicativos de cómo Ilevarla al aula, gestión por parejas, preguntas claves para los estudiantes y posibles extensiones del problema y otras tareas relacionadas. Además, los estudiantes disponen de la solución dada por distintos estudiantes. Estos recursos pueden servir de apoyo para salvar las dificultades a priori que el estudiante del Grado de Primaria puede sentir al abordar estas actividades en relación a su conocimiento matemático. Además, le aportan información sobre el posterior análisis que le planteamos a continuación.

\subsection{Diseño de la tarea previa}

Una tarea escolar es más que simplemente el enunciado de la actividad. Implica toda la "escenografía" que requiere para llevarla a la práctica. A continuación, se les pide a los FMP que conviertan la idea anterior en una tarea para su unidad didáctica, determinando la meta, el contenido, los recursos y materiales y la forma de gestionar la clase (Aguayo, 2018). Es decir, además de la formulación, tienen que completar los demás elementos que describen la tarea relativos a la meta, gestión, materiales, conocimientos previos, actividad matemática y complejidad (Rico y Moreno, 2016). Para ello se seleccionan los elementos del análisis de contenido, cognitivo y de instrucción asociados a la tarea propuesta.

En relación al análisis de contenido, los FMP deben señalar lo relativo a conceptos, procedimientos, representaciones y usos que ya han elaborado en el proceso previo. Por ejemplo, decidir si la tarea la focalizan para trabajar un concepto, como la paridad que aparece en una estrategia de solución, o hacia un procedimiento como realizar descomposiciones de un número en varios sumandos o favorecer el cálculo mental. También analizan el papel de las diferentes representaciones tanto en la formulación como en las respuestas de los estudiantes y dependiendo del tipo de tarea, también se analizan la fenomenología y los usos que aparecen de los contenidos matemáticos. Es cuanto al análisis cognitivo, determinan los objetivos relacionados con la tarea, se revisan las dificultades que podrían darse en la tarea. 
En ese momento, se completan los elementos de análisis de la tarea relativos a identificar la meta de la tarea, determinando de manera precisa la finalidad que se persigue con los estudiantes. Para ello se determinan los conocimientos previos y la actividad matemática que la tarea demanda de los estudiantes, describiendo la gestión de cómo se llevará a cabo en el aula, el agrupamiento y los materiales. Ejemplificamos en la tabla 2 estos elementos de análisis con la propuesta de una estudiante del Grado de Primaria (Rodríguez, 2020).

Tabla 2. Análisis de la tarea extraído de Rodríguez (2020)

\begin{tabular}{ll}
\hline Meta & $\begin{array}{l}\text { Utilizar la descomposición de números y la diferenciación entre pares e } \\
\text { impares en la resolución de problemas. }\end{array}$ \\
\hline $\begin{array}{l}\text { Conocimientos } \\
\text { previos }\end{array}$ & $\begin{array}{l}\text { Utiliza los números naturales y sus relaciones. Conoce las propiedades } \\
\text { de los números naturales y realiza operaciones como sumas, restas, } \\
\text { multiplicaciones y divisiones. Realiza cálculos mentales de operacio- } \\
\text { nes sencillas. }\end{array}$ \\
\hline
\end{tabular}

Materiales Material manipulativo como láminas para que el alumnado pueda realizar los cambios que sean necesarios. Folios. Hoja para escribir las soluciones encontradas.

Gestión En primer lugar, el docente reparte a cada grupo un folio con la actividad a realizar, la cual incluye un primer dibujo a completar y la pregunta en la que se basa el problema (formulación original de NRICH).

Los alumnos comienzan trabajando de manera individual con el objetivo de que investiguen e indaguen en las posibles soluciones. En el caso de que los alumnos se encuentren ante dificultades para comenzar el problema el docente les ofrece algunas pautas como: "intenta poner números en la $\vee$ y luego suma cada brazo. Si no obtienes la misma solución intenta cambiar la posición del algún número para que el total de ambos brazos sea igual".

Tras colocar un número en el vértice el alumno completa el resto de la $\mathrm{V}$, una vez completada una primera $\mathrm{V}$ de manera correcta, el docente les pide que analice detenidamente esas posiciones y busque las posibles relaciones entre los números.

A continuación, se guía a los alumnos para buscar diferentes soluciones. En este momento los alumnos se agrupan en parejas o grupos de tres para que el discutan sobre sus propuestas y se ayuden así en la realización de las actividades.

Actividad matemática
Los estudiantes deben realizar operaciones aritméticas, especialmente sumas y restas. En el caso de utilizar estrategias más allá de analizar casos concretos, pueden reconocer que las sumas de los brazos excluyendo el vértice deben sumar lo mismo. Dado que la suma total de los cinco números es 15 , esto obliga a que el vértice sea un número impar (1, 3 ó 5), puesto que la suma de los dos brazos sin el vértice debe ser par. 


\subsection{Análisis de la riqueza de la tarea}

Una vez realizada la propuesta se plantea analizar la riqueza en relación a los referentes teóricos mostrados, especialmente contrastando los conocimientos previos de la actividad matemática que requiere. Si esta actividad requiere de un contenido nuevo, se reflexiona sobre el modo en el que el estudiante puede alcanzarlo y el papel del maestro para que lo comprenda (a través de preguntas, introduciendo las definiciones o propiedades, utilizando ejemplos, etc.). En este ejemplo, la tarea cumple algunos de los requisitos de riqueza señalados: es compleja, no algorítmica y no rutinaria, permite múltiples estrategias, representaciones y varias soluciones.

El apartado de complejidad es clave para el posterior diseño como tarea de profundización. Esta tarea admite un nivel más bajo pidiendo a los alumnos de primaria que busquen una solución que pueden encontrar por ensayo y error. Se va incrementando la complejidad al pedirles que encuentren todas las soluciones posibles, que justifiquen y comuniquen sus argumentos. Y de mayor complejidad la generalización para valores cualesquiera y otras formas de distribución de los puntos, extendiendo el problema o inventando nuevas versiones (Hernando, 2020). De un modo general, se puede seguir esta secuencia de incremento de complejidad (figura 4).

Figura 4. Secuencia de complejidad

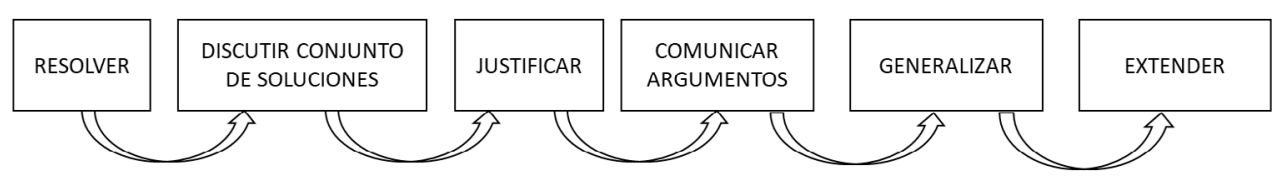

\subsection{Diseño de la tarea de profundización}

Se ha diseñado una tarea rica, que desde sus niveles inferiores de complejidad puede ser propuesta para todo el alumnado. A continuación, se pide a los FPM que la diseñen como tarea de profundización. En este momento es donde se relaciona el análisis de la tarea con las características del talento matemático que se esperan enriquecer. En el caso concreto de esta tarea, se exponen los listados de caracterización del talento de varios autores y se seleccionan los más acordes para la tarea.

En este ejemplo, se consideran los relativos a organizar los datos, localizar la clave de los problemas y la generalización. Se les guía a abordar estas características a través del planteamiento de preguntas asociadas a la actividad matemática. En cursiva aparecen las propias del ejemplo seleccionado.

- Resolución. Observa una de las soluciones. ¿Puedes obtener otra solución distinta intercambiando dos de los números? ¿Qué pasa si intercambiamos dos círculos del mismo brazo distintos del vértice? ¿Y si intercambiamos los dos brazos? ¿Es el vértice un círculo clave para nuestro problema? ¿Por qué?

- Justificación. ¿Para qué valores del vértice has encontrado solución? ¿Tienen algo en común? ¿Por qué no puedes encontrar soluciones en otros casos? Organiza toda esta información en una tabla. 
- Generalización. ¿Valen tus argumentos para cualesquiera cinco números consecutivos? ¿Podemos encontrar una estrategia sencilla para sumarlos? ¿Cuándo la suma de cinco números consecutivos es par?

- Extensión. ¿Qué ocurre si en vez de una $V$ es una $L$ o una X? ¿Qué cambia en el problema? ¿Y con otro tipo de letras? ¿Son tus argumentos válidos en estas situaciones? Inventa un nuevo problema a partir de lo que has aprendido en esta tarea. ¿Se te ocurre relacionarlo con alguna situación de la vida cotidiana?

La gestión se centra en plantear las preguntas para que las aborden de manera individual, luego hagan puestas en común en grupos reducidos y consensuen una respuesta de grupo. En la puesta en común, se recogen sus ideas y se les pide que las argumenten.

Retomando la tarea formativa, una vez que cada FMP ha diseñado su tarea de profundización, se les pide que la valoren de acuerdo a criterios de calidad asociados al proceso seguido: ¿Qué contenidos matemáticos se enriquecen? ¿Qué elementos de razonamiento matemático se favorecen? ¿Qué características del talento se estimulan? ¿Por qué es una tarea de profundización?

\section{Conclusión}

El diseño de programas de profundización es una necesidad que se ha puesto de manifiesto en recientes normativas curriculares (por ejemplo, Junta de Andalucía, 2021). En este trabajo se ha propuesto una tarea formativa para que el FMP aborde esta problemática desde la asignatura de Diseño y desarrollo del currículum en Educación Primaria, donde uno de los objetivos es diseñar espacios de aprendizaje en contextos de diversidad (Ramírez, 2021).

Consideramos que el análisis didáctico aporta herramientas fundamentadas para que el FMP profundice tanto en el conocimiento del contenido matemático como en el didáctico (Rico, 2015). Al considerar la riqueza en el análisis de las tareas, se pone el foco en elementos que requieren que el estudiante ponga en juego sus conocimientos matemáticos. El partir de ideas ricas seleccionadas de autores reconocidos para una temática específica, y de las que disponen de soluciones y orientaciones metodológicas para llevarlas a cabo, puede ayudarles a salvar posibles debilidades en sus propios conocimientos matemáticos. La comprensión de las características asociadas al talento matemático y el proceso formativo propuesto establece unas pautas que pueden servir de referencia para atender a las necesidades educativas especiales del futuro alumnado al que deberán atender.

Enfatizamos que en la formación del FMP se les presenten fuentes de ideas ricas para no partir de cero en el proceso de creación de sus propias propuestas, además de orientarles con un proceso sistemático y fundamentado que les ayude en el diseño de la planificación. Además, valoramos que su propio proceso formativo se base en tareas formativas que ejemplifiquen la idea que se les quiere transmitir de enseñanza a través de tareas ricas. 


\section{Agradecimientos}

El presente trabajo de investigación se ha realizado dentro del proyecto de investigación del Plan Nacional I+D con referencia Edu2017-84377-R, financiado por el Ministerio de Economía y Competitividad de España. También forma parte del proyecto de la Fundación Española para la Ciencia y la Tecnología (FECYT) de la convocatoria de ayudas para el fomento de la cultura científica, tecnológica y de la innovación con referencia FCT-19-14644 (Ministerio de Ciencia e Innovación).

\section{REFERENCIAS BIBLIOGRÁFICAS}

Aguayo, C. (2018). El análisis didáctico en la formación inicial de maestros de primaria [Tesis doctoral sin publicar]. Universidad de Granada.

Ball, D. L., Thames, M. H. y Phelps, G. (2008). Content knowledge for teaching: What makes it special? Journal of Teacher Education, 59(5), 389-407. https://doi. org/10.1177/0022487108324554

Blanco, R., Ríos, C. y Benavides, M. (2004). Respuesta educativa para los niños con talento. En M. Benavides, A. Maz, E. Castro y R. Blanco (Eds.), La Educación de niños con talento en Iberoamérica (pp. 49-60). OREALC-Unesco.

Burkhart, H. y Swan, M. (2013). Task design for systemic improvement: Principles and frameworks. En C. Margolinas (Ed.), Task design in mathematics education: Proceedings of ICMI Study 22 (pp. 433- 432). ICME.

Clarke, B., Grevholm, B. y Millman, R. (2009). Tasks in primary mathematics teacher education. Purpose, use and exemplars. Springer.

Flores, P. y Rico, L. (Coords.) (2015). Enseñanza y aprendizaje de las matemáticas en Educación Primaria. Pirámide.

Freiman, V. (2006). Problems to discover and to boost mathematical talent in early grades: A challenging situations approach. Mathematics Enthusiast, 3(1), 51-75.

Gómez, P. (2007). Desarrollo del conocimiento didáctico en un plan de formación inicial de profesores de matemáticas de secundaria [Tesis doctoral]. Universidad de Granada.

Good, S. (2013). What is a rich task? Greater Cleveland Council of Teachers of Mathematics. http://mygcctmonline.org/resources/19sept2013/Rich_Tasks-What_ Where_Why_How.pdf

Greenes, C. (1981). Identifying the gifted student in mathematics. Arithmetic Teacher, 28(8), 14-17.

Grootenboer, P. (2009). Rich mathematical tasks in the Maths in the Kimberley (MITK) Project. En R. Hunter, B. Bicknell y T. Burgess (Eds.), Crossing divides, Proceedings of the 32and annual conference of the MERGA, 1 (pp. 696-699). MERGA.

Hernando, E. (2020). Visión retrospectiva y extensión del problema. SUMA: Revista sobre Enseñanza y Aprendizaje de las Matemáticas, 94, 49-58.

Junta de Andalucía (2021). Orden de 15 de enero de 2021, por la que se desarrolla el currículo correspondiente a la etapa de Educación Primaria en la Comunidad Autónoma de Andalucía, se regulan determinados aspectos de la atención a la diversidad, se establece la ordenación de la evaluación del proceso de aprendi- 
zaje del alumnado y se determina el proceso de tránsito entre distintas etapas educativas. Consejería de Educación y Deporte de la Junta de Andalucía.

Krutetskii, V. A. (1976). The psychology of Mathematical Abilities in Schoolchildren. University of Chicago Press.

Liljedahl, P., Chernoff, E. y Zazkis, R. (2007). Interweaving mathematics and pedagogy in task design: A tale of one task. Journal of Mathematics Teacher Education, 10(4-6), 239-249. https://doi.org/10.1007/s10857-007-9047-7

Lingard, R. Ladwig, J., Mills, M., Bahr, M., Chant, D., Warry, M., Allwood, J., Capeness, R., Christie, P., Gore, J., Haydes, D. y Luke, A. (2001). The Queensland school reform longitudinal study. Education Queensland.

Miller, R. C. (1990). Discovering mathematical talent. ERIC Digest E482. Office of Educational Research and Improvement.

Niederer, K., Irwin, R. C., Irwin, K. C. y Reilly, I. L. (2003). Identification of mathematically gifted children in New Zealand. High Ability Studies, 14(1), 71-84. https:// doi.org/10.1080/13598130304088

Piñeiro, J. L., Ramírez, R. y Segovia, I. (2017). Detección del talento matemático en Educación Infantil. Edma 0-6: Educación Matemática en la Infancia, 6(2), 56-71.

Ramírez, R. (2021). Proyecto docente para la plaza de Profesor Titular del Área de Didáctica de las Matemáticas en el Grado de Maestro en Educación Primaria en la Materia Diseño y Desarrollo del currículum en Educación Primaria. Universidad de Granada. https://digibug.ugr.es/handle/10481/65919

Ramírez, R., Castro-Rodríguez, E., Piñeiro, J. L. y Ruiz-Hidalgo, J. F. (2018). What makes a task a problem in early childhood education? European Early childhood Education Research Journal, 26(4), 574-588.

Rico, L. (Ed.) (1997a). Bases teóricas del currículo de matemáticas en educación secundaria. Síntesis.

Rico, L. (Ed.). (1997b). Educación matemática en la enseñanza secundaria. Ice-Horsori.

Rico, L. (2015). Matemáticas escolares y conocimiento didáctico. En P. Flores y L. Rico (Coords.), Enseñanza y aprendizaje de las matemáticas en educación primaria (pp. 21-40). Pirámide.

Rico, L. (2016). Matemáticas y análisis didáctico. En L. Rico y A. Moreno (Coords.), Elementos de didáctica de la matemática para el profesor de secundaria (pp. 85100). Pirámide.

Rico, L., Lupiáñez, J. L. y Molina., M. (Eds.) (2013). El análisis didáctico en educación matemática. Metodología de investigación, formación de profesores e innovación curricular. Comares.

Rico, L. y Moreno, A. (Coords.) (2016). Elementos de didáctica de la matemática para el profesor de secundaria. Pirámide.

Rodríguez, M. (2020). Organización de datos, justificación, generalización y extensión en tareas de enriquecimiento matemático. Trabajo fin de Grado de Primaria de la Universidad de Granada.

Ruiz-Hidalgo, J. F., Flores, P., Ramírez, R. y Fernández-Plaza, J. A. (2019). Tareas que desarrollan el sentido matemático en la formación inicial de profesores. Educación Matemática, 31(1), 121-143. 
Stein, M. K. y Smith, M. S. (1998). Mathematical tasks as a framework for reflection: From research to practice. Mathematics Teaching in the Middle School, 3(4), 268-275.

Stein, M. K., Smith, M, Henningsen, M. y Silver, E. (2000). Implementing StandardsBased Mathematics Instruction. Teachers College Press.

Tirosh, D. y Woods, T. (2008). The international handbook of mathematics teacher education (Vol. 2). Sense Publishers. 